\title{
Proposal for a loophole-free Bell test using homodyne detection
}

\author{
R. García-Patrón Sánchez, ${ }^{1}$ J. Fiurášek, ${ }^{1,2}$ N.J. Cerf, ${ }^{1}$ J. Wenger ${ }^{3}$ R. Tualle-Brouri, ${ }^{3}$ and Ph. Grangier ${ }^{3}$ \\ ${ }^{1}$ QUIC, Ecole Polytechnique, CP 165, Université Libre de Bruxelles, 1050 Bruxelles, Belgium \\ ${ }^{2}$ Department of Optics, Palacký University, 17. listopadu 50, 77200 Olomouc, Czech Republic \\ ${ }^{3}$ Laboratoire Charles Fabry de l'Institut d'Optique, CNRS UMR 8501, F-91403 Orsay, France
}

\begin{abstract}
We propose a feasible optical setup allowing for a loophole-free Bell test with efficient homodyne detection. A non-gaussian entangled state is generated from a two-mode squeezed vacuum by subtracting a single photon from each mode, using beamsplitters and standard low-efficiency singlephoton detectors. A Bell violation exceeding $1 \%$ is achievable with $6 \mathrm{~dB}$ squeezed light and an homodyne efficiency around 95\%. A detailed feasibility analysis, based upon the recent generation of single-mode non-gaussian states, confirms that this method opens a promising avenue towards a complete experimental Bell test.
\end{abstract}

PACS numbers: $\quad$ 03.65.Ud, 03.67.-a, 42.50.Dv

In their seminal 1935 paper, Einstein, Podolsky, and Rosen (EPR) advocated that if "local realism" is taken for granted, then quantum theory is an incomplete description of the physical world [1]. The EPR argument gained a renewed attention in 1964 when John Bell derived his famous inequalities, which must be satisfied within the framework of any local realistic theory [2]. The violation of Bell inequalities (BI), predicted by quantum mechanics, has since then been observed in many experiments $3,4,4,5,6,6]$, disproving the concept of local realism. So far, however, all these tests suffered from "loopholes" allowing a local realistic explanation of the experimental observations by exploiting either the low detector efficiency 8] or the time-like interval between the two detection events [9]. In this Letter, we propose a realistic experimental scheme based on the conditional generation of non-gaussian entangled light states and balanced homodyning, which can circumvent these difficulties and allows for a "loophole-free" experimental Bell test.

A test of Bell inequality violation typically involves two distant parties, Alice and Bob, who simultaneously carry out measurements on two systems prepared in an entangled quantum state. The measurement events (including the measurement choice) at Alice's and Bob's sites must be spacelike separated in order to avoid the locality loophole, that is, to rule out any possible communication between the two parties. Optical systems are therefore particularly suitable candidates for Bell tests, because entangled photon pairs can now be generated and distributed over long distances [5, 6 . . However, the currently available single-photon detectors suffer from too low efficiencies $\eta$, opening the so-called detector-efficiency loophole, that is, the experimental data can be explained by local realistic theories wherein the detectors only click with probability $\eta$. This loophole is present in all optical BI tests today.

In contrast, very high detection efficiencies can be reached in optical systems using balanced homodyne detectors 10, 11, 12], opening a very promising alternative to Bell tests based on single-photon detectors. Several theoretical works have shown that a violation of BI may indeed be observed with balanced homodyning provided that some very specific entangled light states can be prepared [13, 14, 15]. Although a violation up to the maximum theoretical limit can be achieved with homodyne detection 15], all the states required in 13, 14, 15. unfortunately appear to be experimentally infeasible. On the other hand, the experimentally accessible 16, 17, 18 twomode squeezed vacuum states are unsuitable for a Bell test because they are characterized by a positive-definite gaussian Wigner function, which provides an explicit hidden variable model for homodyne measurements. So far, no feasible experimental scheme has been found that could be used to prepare non-gaussian states that exhibit a violation of $\mathrm{BI}$ with balanced homodyning.

In this Letter, we show that a strikingly simple optical setup (see Fig. 1) can be used to conditionally generate non-gaussian states that are suitable for this purpose. Our scheme requires a pulsed source of two-mode squeezed vacuum state, which can be expressed in the Fock basis as

$$
\left|\psi_{\text {in }}\right\rangle_{A B}=\sqrt{1-\lambda^{2}} \sum_{n=0}^{\infty} \lambda^{n}|n, n\rangle_{A B}
$$

where $\lambda=\tanh (r)$ and $r$ is the squeezing constant. The basic idea is to "degaussify" this state by subtracting a photon from each mode [19, 20, 21]. More precisely, we produce a non-gaussian entangled state with the use of two unbalanced beam splitters $\mathrm{BS}_{A}$ and $\mathrm{BS}_{B}$ with intensity transmittance $T$, followed by two photon-counting detectors $\mathrm{PD}_{A}$ and $\mathrm{PD}_{B}$ such as avalanche photodiodes (APD). The successful state preparation is heralded by a click of both $\mathrm{PD}_{A}$ and $\mathrm{PD}_{B}$. We shall see that a detector efficiency as low as $\eta=10 \%$ suffices for the preparation of states exhibiting a violation of BI. Very recently, the generation of pulsed single-mode non-gaussian states by photon subtraction from squeezed states has been demonstrated experimentally by some of us 22]. We therefore can realistically envision the experimental realization of 


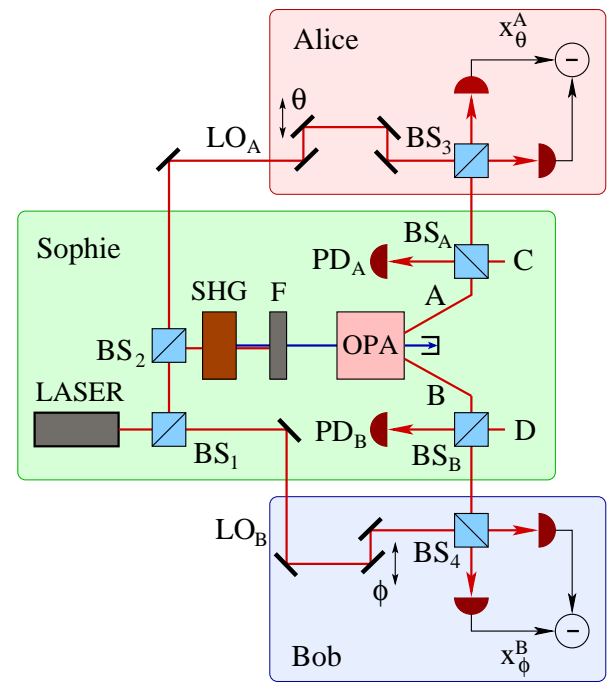

FIG. 1: Proposed experimental setup. The source (controlled by Sophie) is based on a master laser beam, which is converted into second harmonic in a nonlinear crystal (SHG). After spectral filtering (F), the second harmonic beam pumps an optical parametric amplifier (OPA) which generates twomode squeezed vacuum in modes A and B. Single photons are conditionally subtracted from modes $\mathrm{A}$ and $\mathrm{B}$ with the use of the beam splitters $\mathrm{BS}_{A}$ and $\mathrm{BS}_{B}$ and single-photon detectors $\mathrm{PD}_{A}$ and $\mathrm{PD}_{B}$. Alice (Bob) measures a quadrature of mode A (B) using a balanced homodyne detector that consists of a balanced beam splitter $\mathrm{BS}_{3}\left(\mathrm{BS}_{4}\right)$ and a pair of highlyefficient photodiodes. The local oscillators $\mathrm{LO}_{A}$ and $\mathrm{LO}_{B}$ are extracted from the laser beam by means of two additional beam splitters $\mathrm{BS}_{1}$ and $\mathrm{BS}_{2}$.

a Bell test with non-gaussian two-mode states prepared along the same lines.

The photon subtraction can easily be understood in the limit of high transmittance of $\mathrm{BS}_{A}$ and $\mathrm{BS}_{B}(T \rightarrow 1)$, since then the most probable event leading to a click at each PD is when a single photon is reflected by each BS. In this limit, the conditionally generated state is very close to a pure state 20]

$$
\left|\psi_{\text {out }}\right\rangle_{A B} \propto \hat{a}_{A} \hat{a}_{B}\left|\psi_{\text {in }}\right\rangle_{A B} \propto \sum_{n=0}^{\infty}(n+1)(T \lambda)^{n}|n, n\rangle_{A B}
$$

where $\hat{a}_{A, B}$ are annihilation operators. This state is nongaussian, and therefore suitable for a Bell test based on homodyning.

In the experiment proposed here, Alice and Bob measure the quadratures $x_{\theta}^{A}=\cos \theta x^{A}+\sin \theta p^{A}$ and $x_{\phi}^{B}=$ $\cos \phi x^{B}+\sin \phi p^{B}$, which have continuous spectra and satisfy $\left[x^{j}, p^{k}\right]=i \delta_{j k}$. In order to use the standard Bell-CHSH inequality [23], we discretize these quadratures by postulating that the outcome is +1 when $x \geq 0$, and -1 otherwise. Alice and Bob must then choose randomly and independently between two different measurements, corresponding to the choices of two angles $\theta_{1}, \theta_{2}$ and $\phi_{1}, \phi_{2}$. Their measurements outcomes are thus described by four variables $a_{1}, a_{2}, b_{1}, b_{2}$, with values +1 or -1 . One defines the usual Bell parameter

$$
S=\left\langle a_{1} b_{1}\right\rangle+\left\langle a_{1} b_{2}\right\rangle+\left\langle a_{2} b_{1}\right\rangle-\left\langle a_{2} b_{2}\right\rangle,
$$

where $\left\langle a_{j} b_{k}\right\rangle$ denotes the average over the subset of the experimental data when Alice measured $a_{j}$ and, simultaneously, Bob measured $b_{k}$. As we shall see, exploiting the fact that $\mathrm{PD}_{A}$ and $\mathrm{PD}_{B}$ can be viewed here as "eventready" detectors 24], one can prove that all local-realistic models for Alice and Bob measurements must satisfy the Bell-CHSH inequality $|S| \leq 2[23]$.

In the formalism of "event-ready" detectors introduced by John Bell 24], one should know, by some initiating event, when a measurable system has been produced. The main idea is to pre-select - rather than post-select - the relevant events. For that purpose, one considers 3 partners, Alice and Bob who perform the measurements, and Sophie who controls the source, see Fig. 1. The entire data analysis must then be performed on a pulsed basis, with Sophie sending time-tagged light pulses (local oscillator and squeezed light) to Alice and Bob. In each experimental run, Sophie records whether her photodetectors clicked, while Alice and Bob carry out measurements of one of two randomly chosen quadratures. After registering a large number of events, the three partners discard all events not corresponding to an "event-ready" doubleclick registered by Sophie. The correlation coefficients $\left\langle a_{j} b_{k}\right\rangle$ are then evaluated from all remaining events, and plugged into the $S$ parameter (3).

In a local realistic approach, the light pulses in each time slot carry some random unknown parameters $\mu$, which will ultimately determine the sign of $x_{\theta}^{A}$ and $x_{\phi}^{B}$. The crucial feature is that the experiment should be designed in such a way that the measurements at Alice's and Bob's sites, as well as the detection of clicks from the conditioning detectors by Sophie, are all spacelike separated [25]. Therefore, the probability distribution $p(\mu)$ must be independent of the measurement phases $\theta_{1,2}$ and $\phi_{1,2}$. The measured sign $s_{A}$ on Alice's side (resp. $s_{B}$ on Bob's side) only depends on $\mu$ and $\theta$ (resp. $\phi$ on Bob's side). One can thus write $\left\langle a_{j} b_{k}\right\rangle=\int d \mu p(\mu) s_{A}\left(\theta_{j}, \mu\right) s_{B}\left(\phi_{k}, \mu\right)$, from which the derivation of the Bell-CHSH inequality is very standard 23]. The only requirement needed in the above reasoning is local realism, the absence of any other assumption being the condition for a "loophole-free" Bell test.

Let us now evaluate the quantum mechanical value of the $S$ parameter in the proposed experimental scheme. We only briefly outline the main steps of the calculation, the details will be presented elsewhere 26]. We model realistic photodetectors (which have a limited quantum efficiency $\eta<1$, and cannot discriminate between one and multiple photon detection events) as ideal detectors preceded with a "virtual" beam splitter of transmittance $\eta$. Ideal detectors respond with two different outcomes, 
either a no-click (projection onto the vacuum) or a click (projection on all states with at least one photon). Similarly, a balanced homodyne detector with efficiency $\eta_{\mathrm{BHD}}$ is modeled as a perfect homodyne detector preceded with a "virtual" beam splitter of transmittance $\eta_{\mathrm{BHD}}$. At the output of the OPA, the modes A and B are prepared in a two-mode squeezed vacuum state, and the auxiliary modes $\mathrm{C}$ and $\mathrm{D}$ are in vacuum state. The Wigner function of the state of modes ABCD is a gaussian function centered at the origin,

$$
W_{\mathrm{in}, A B C D}(r)=\frac{1}{\pi^{4} \sqrt{\operatorname{det} \gamma_{\mathrm{in}}}} \exp \left[-r^{T} \gamma_{\mathrm{in}}^{-1} r\right] .
$$

This state is fully characterized by the covariance matrix $\gamma_{i j}=\left\langle r_{i} r_{j}+r_{j} r_{i}\right\rangle-2\left\langle r_{i}\right\rangle\left\langle r_{j}\right\rangle$, where $r=$ $\left(x^{A}, p^{A}, \ldots, x^{D}, p^{D}\right)$ is a vector of quadrature components. The input covariance matrix is $\gamma_{\mathrm{in}}=\gamma_{\mathrm{in}, A B} \oplus I_{C D}$, where $I$ is the identity matrix and $\gamma_{\mathrm{in}, A B}$ denotes the covariance matrix of the two-mode squeezed vacuum (11). After combining modes $\mathrm{A}$ and $\mathrm{C}(\mathrm{B}$ and $\mathrm{D})$ on an unbalanced beam splitter $\mathrm{BS}_{A}\left(\mathrm{BS}_{B}\right)$ with transmittance $T$, the modes $\mathrm{C}$ and $\mathrm{D}$ are detected by the photoncounting detectors $\mathrm{PD}_{A}$ and $\mathrm{PD}_{B}$, while modes $\mathrm{A}$ and $\mathrm{B}$ are sent to the homodyne detectors. The covariance matrix $\gamma_{\text {out }}$ of the mixed gaussian state of modes $\mathrm{ABCD}$ after passing through the beam splitters $\mathrm{BS}_{A}$ and $\mathrm{BS}_{B}$ and the four "virtual" beam splitters modeling the imperfect detectors is related to $\gamma_{\text {in }}$ via a gaussian completely positive map [27], $\gamma_{\text {out }}=S_{\eta} S_{\mathrm{BS}} \gamma_{\mathrm{in}} S_{\mathrm{BS}}^{T} S_{\eta}^{T}+G$, where $S_{\mathrm{BS}}$ describes the mode coupling in $\mathrm{BS}_{A}$ and $\mathrm{BS}_{B}$, and the matrices $S_{\eta}=\sqrt{\eta_{\mathrm{BHD}}} I_{A B} \oplus \sqrt{\eta} I_{C D}$ and $G=\left(1-\eta_{\mathrm{BHD}}\right) I_{A B} \oplus(1-\eta) I_{C D}$ account for the imperfect detectors.

The Wigner function of the state of modes A and B prepared by conditioning on observing clicks at $\mathrm{PD}_{A}$ and $\mathrm{PD}_{B}$ can then be expressed as a linear combination of four gaussian functions,

$$
W(r)=\frac{1}{\pi^{2} P \sqrt{\operatorname{det} \gamma_{\text {out }}}} \sum_{j=1}^{4} \frac{q_{j}}{\sqrt{\operatorname{det} \Gamma_{j, C D}}} \exp \left[-r^{T} \Gamma_{j} r\right],
$$

where $P=\left(\operatorname{det} \gamma_{\text {out }}\right)^{-1 / 2} \sum_{j=1}^{4} q_{j}\left[\operatorname{det}\left(\Gamma_{j} \Gamma_{j, C D}\right)\right]^{-1 / 2}$ stands for the probability of success, $r=$ $\left(x^{A}, p^{A}, x^{B}, p^{B}\right)$, and we have defined $q_{1}=1$, $q_{2}=q_{3}=-2, q_{4}=4$. The various matrices appearing in Eq. (5) are obtained from $\gamma_{\text {out }}^{-1}$, which can be split into four smaller submatrices as $\gamma_{\text {out }}^{-1}=\left[\begin{array}{cc}\Gamma_{A B} & \sigma \\ \sigma^{T} & \Gamma_{C D}\end{array}\right]$. These four submatrices are then used to define $\Gamma_{j}=\Gamma_{A B}-\sigma \Gamma_{j, C D}^{-1} \sigma^{T}$, where $\Gamma_{1, C D}=\Gamma_{C D}$, $\Gamma_{2, C D}=\Gamma_{C D}+I_{C} \oplus 0_{D}, \Gamma_{3, C D}=\Gamma_{C D}+0_{C} \oplus I_{D}$, and $\Gamma_{4, C D}=\Gamma_{C D}+I_{C D}$

After discretization of the quadratures, the correlation coefficient $E\left(\theta_{j}, \phi_{k}\right) \equiv\left\langle a_{j} b_{k}\right\rangle$ can be expressed as

$$
E\left(\theta_{j}, \phi_{k}\right)=\int_{-\infty}^{\infty} \operatorname{sign}\left(x_{\theta_{j}}^{A} x_{\phi_{k}}^{B}\right) P\left(x_{\theta_{j}}^{A}, x_{\phi_{k}}^{B}\right) d x_{\theta_{j}}^{A} d x_{\phi_{k}}^{B},
$$
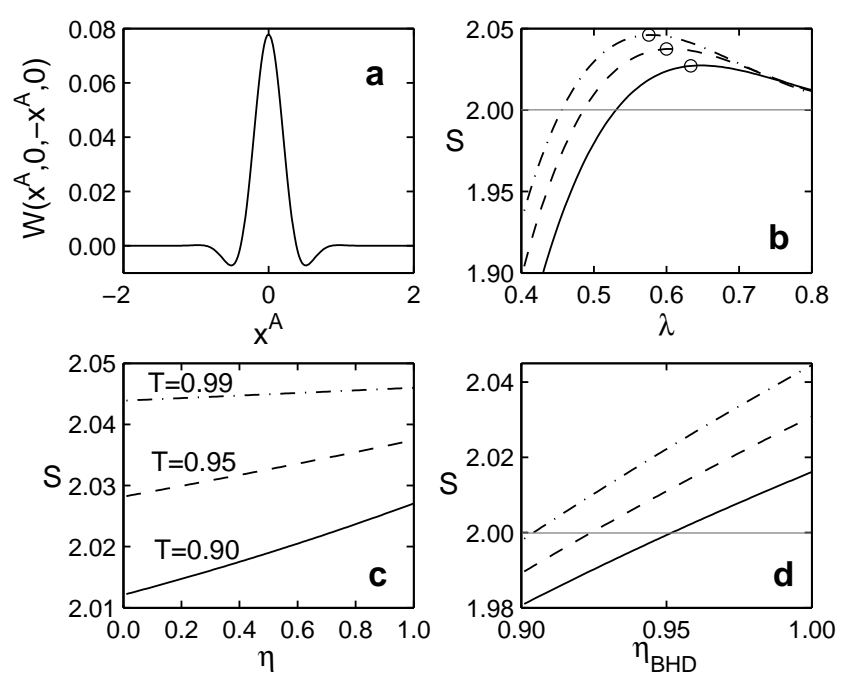

FIG. 2: Violation of Bell-CHSH inequality with the conditionally-prepared non-gaussian state. (a) Onedimensional cut of the Wigner function of the conditionally generated non-gaussian two-mode state (with $\lambda=0.5, T=$ 0.95 , and $\eta=30 \%$ ) along the line $x^{B}=-x^{A}, p^{A}=p^{B}=0$. Notice the regions where $W$ is negative. (b) Bell parameter $S$ as a function of the squeezing $\lambda$ of the initial two-mode squeezed vacuum. The curves are plotted for perfect detectors $\left(\eta=\eta_{\mathrm{BHD}}=100 \%\right)$ with $T=0.9$ (solid line), $T=0.95$ (dashed line), and $T=0.99$ (dot-dashed line). The open circles mark the points where $T \lambda=0.57$. (c) Bell parameter $S$ as a function of the efficiency $\eta$ of the single-photon detectors, for $\lambda T=0.57, \eta_{\mathrm{BHD}}=100 \%$, and the same three $T$ 's as in (b). (d) Bell parameter $S$ as a function of the efficiency $\eta_{\mathrm{BHD}}$ of the balanced homodyne detectors, for $\lambda T=0.57, \eta=30 \%$, and the same three $T$ 's as in (b).

where $P\left(x_{\theta_{j}}^{A}, x_{\phi_{k}}^{B}\right)$ is the joint probability distribution of the two commuting quadratures $x_{\theta_{j}}^{A}$ and $x_{\phi_{k}}^{B}$, which can be determined as a marginal distribution from the Wigner function given by Eq. (15). The correlation coefficient $E$ (and therefore $S$ ) can then be expressed in a closed form by analytically integrating the resulting gaussian functions.

The results plotted in Fig. 2 were obtained for the optimal choice of angles $\theta_{1}=0, \theta_{2}=\pi / 2, \phi_{1}=-\pi / 4$, $\phi_{2}=\pi / 4$. Figure $2(\mathrm{a})$ shows that the Wigner function $W$ is negative in some regions of the phase space, which is a necessary condition for the observation of a violation of BI with homodyne detectors. Fig. 2(b) illustrates that the Bell inequality $|S| \leq 2$ can indeed be violated with the proposed set-up, and shows that there is an optimal squeezing $\lambda_{\text {opt }}$ which maximizes $S$. A simplified calculation assuming perfect detectors with single-photon resolution [26] predicts that $\lambda_{\mathrm{opt}} T \approx 0.57$. It follows from Fig. 2(c) that the Bell factor $S$ depends only very weakly on the efficiency $\eta$ of the single-photon detectors, so the Bell inequality can be violated even if $\eta<10 \%$. In contrast, the efficiency of the homodyne detectors $\eta_{\mathrm{BHD}}$ must 
be above $\sim 90 \%$, see Fig. $2(\mathrm{~d})$.

The maximum Bell factor achievable with our scheme is about $S_{\max } \approx 2.046$ which represents a BI violation of $2.3 \%$. This is a small, but statistically significant violation, which should be possible to observe experimentally. The required degree of squeezing to get close to $S_{\max }$ corresponds to $\lambda \approx 0.57$, i.e., approximately $5.6 \mathrm{~dB}$, a value that has already been achieved experimentally [10, 28]. Another important parameter is the transmittance $T$ of the beam splitters $\mathrm{BS}_{A}$ and $\mathrm{BS}_{B}$, which must be as high as possible for maximizing $S$. However, the probability of successful state preparation can be estimated as $P \approx \eta^{2}(1-T)^{2}$, so that it quickly drops when $T$ approaches unity. Thus there is a tradeoff between $S$ and $P$ which needs to be optimized by taking into account the statistical uncertainties of the data.

In order to be more specific, let us consider the singlemode version of our scheme, which has already been implemented experimentally [22]. It is based on a commercial cavity-dumped titanium-sapphire laser, delivering nearly Fourier-limited pulses at $850 \mathrm{~nm}$, with a duration of $150 \mathrm{fs}$ and a repetition rate of $790 \mathrm{kHz}$. Squeezed vacuum pulses, generated by parametric deamplification, are sent through a beam splitter, and the reflected beam is detected by a silicon APD. Conditional on observing a click, the transmitted pulse is prepared in a singlemode non-gaussian state, which is measured by homodyne detector with overall efficiency $\eta_{\mathrm{BHD}} \approx 75 \%$. This experiment gives us useful estimates for a possible Bell test. First, the delay between pulses $(1.2 \mu \mathrm{s})$ allows ample time for individual pulse analysis. A fast random choice of the analyzed quadratures can be performed using electro-optical modulators on the LO beams, triggered for instance by digitizing the shot-noise of locally generated auxiliary beams. Switching times around 100 ns, associated with propagation distances of a few tens of meters, seem quite feasible. The APDs can be triggered only when a pulse is expected, reducing the effect of dark counts to a negligible value. The intrinsic APD efficiency is about $50 \%$, but the filtering used to select a single mode currently reduces the overall $\eta$ to less than $5 \%$, which should be improved for accumulating enough statistics.

We can thus define a set of realistic parameter values that should be reached in a loophole-free Bell test : with $\eta=30 \%, T=95 \%$, and $\lambda=0.6, \mathrm{BI}$ are violated by about $1 \%$ if the homodyne efficiency $\eta_{\mathrm{BHD}}$ is larger than $95 \%$ (see Fig. 2(d)). With a repetition rate of $1 \mathrm{MHz}$ and $P \approx 2.6 \times 10^{-4}$, the number of data samples would be several hundreds per second, so that the required statistics to see a violation in the percent range could be obtained in a reasonable time (less than one hour). In addition, the electronic noise of the homodyne detectors should be 15-20 dB below shot noise, attainable with low-noise charge amplifiers. All these numbers have already been reached separately in various experiments, but attaining them simultaneously certainly represents a serious challenge. Nevertheless, taking into account many possible experimental improvements, the existence of an experimental window for a loophole-free test of Bell inequalities can be considered as highly plausible. As a conclusion, it appears that, with quantum continuous variables, a reasonable compromise can be found between the experimental constraints and the very stringent requirements imposed by a loophole-free test of Bell inequalities.

This work is supported by the European IST/FET program, the Communauté Française de Belgique under Grant No. ARC 00/05-251, and the IUAP programme of the Belgian government under Grant No. V-18. JF also acknowledges support from the Grant No. LN00A015 of the Czech Ministry of Education.

[1] A. Einstein, B. Podolsky, and N. Rosen, Phys. Rev. 47, 777 (1935).

[2] J.S. Bell, Physics (Long Island City, N.Y.) 1, 195 (1964).

[3] S.J. Freedman and J.F. Clauser, Phys. Rev. Lett. 28, 938 (1972).

[4] A. Aspect, P. Grangier, and G. Roger, Phys. Rev. Lett. 47, 460 (1981); ibid., 49, 91 (1982); A. Aspect, J. Dalibard, and G. Roger, Phys. Rev. Lett. 49, 1804 (1982).

[5] G. Weihs et al., Phys. Rev. Lett. 81, 5039 (1998).

[6] W. Tittel et al., Phys. Rev. A 57, 3229 (1998).

[7] M.A. Rowe et al., Nature 409, 791 (2001).

[8] Philip M. Pearle, Phys. Rev. D 2, 1418 (1970).

[9] E. Santos, Phys. Rev. A 46, 3646 (1992).

[10] E.S. Polzik, J. Carri, and H.J. Kimble, Phys. Rev. Lett. 68, 3020 (1992).

[11] T.C. Zhang et al., Phys. Rev. A 67, 033802 (2003).

[12] F. Grosshans et al., Nature (London) 421, 238 (2003).

[13] A. Gilchrist, P. Deuar, and M.D. Reid, Phys. Rev. Lett. 80, 3169 (1998).

[14] W.J. Munro, Phys. Rev. A 59, 4197 (1999).

[15] J. Wenger et al., Phys. Rev. A 67, 012105 (2003).

16] Z.Y. Ou et al., Phys. Rev. Lett. 68, 3663 (1992).

[17] C. Schori, J.L. Sørensen, and E.S. Polzik, Phys. Rev. A 66, 033802 (2002).

[18] W.P. Bowen et al., Phys. Rev. A 69, 012304 (2004).

[19] T. Opatrný, G. Kurizki, and D.-G. Welsch, Phys. Rev. A 61, 032302 (2000).

[20] P.T. Cochrane, T.C. Ralph, and G.J. Milburn, Phys. Rev. A 65, 062306 (2002).

[21] S. Olivares, M.G.A. Paris, and R. Bonifacio, Phys. Rev. A 67, 032314 (2003).

[22] J. Wenger, R. Tualle-Brouri, and P. Grangier, quant-ph/0402192 to appear in Phys. Rev. Lett. (2004).

[23] J.F. Clauser et al., Phys. Rev. Lett. 23, 880 (1969)

[24] J.S. Bell, Speakable and Unspeakable in Quantum Mechanics (Cambridge University Press, 1988) p. 105.

[25] A. Aspect, Phys. Rev. D 14, 1944 (1976).

[26] R. García-Patrón Sánchez, J. Fiurášek, and N.J. Cerf, in preparation.

[27] J. Eisert and M.B. Plenio, Phys. Rev. Lett. 89, 097901 (2002); J. Fiurášek, Phys. Rev. A 66, 012304 (2002).

[28] P.K. Lam et al., J. Opt. B: Quantum Semiclass. Opt. 1, 469 (1999). 